\title{
EDITORIAL \\ Constitución digital: Documento para el debate constituyente en Chile
}

\author{
Centro de Estudios en Derecho Informático ${ }^{1}$ \\ Universidad de Chile
}

$\mathrm{El} 4$ de julio de 2021 se instalará la Convención Constitucional que tiene por mandato redactar y aprobar una nueva constitución política para Chile. ${ }^{2}$ Ese día, 155 convencionales constituyentes aceptarán el cargo, sesionarán e iniciarán este proceso histórico para el país, que es la respuesta institucional al estallido social de octubre de 2019.

En este contexto, como Centro de Estudios en Derecho Informático de la Facultad de Derecho de la Universidad de Chile queremos contribuir al proceso constituyente desde nuestra especialidad, promoviendo un debate público, abierto y transparente respecto a aquellos aspectos que resultan fundamentales para las personas y las comunidades en la sociedad digital. Entre estos aspectos podemos destacar las futuras reglas constitucionales sobre el ejercicio de derechos y libertades en los entornos digitales, la igualdad ante la ley, la no discriminación, la relación de las personas con el Estado y el resguardo de otros intereses jurídicamente relevantes.

Con ese objetivo hemos elaborado una propuesta inicial que ofrece una nueva mirada de ciertos derechos y libertades fundamentales tradicionales cuyo enfoque o formulación pueden verse restringidos, afectados o potenciados a partir del uso cada vez más intensivo de tecnologías digitales y, eventualmente, la adición de nuevos derechos, que surgen y se explican a partir de esa nueva realidad social, cultural y tecnológica. Además, esta propuesta contiene algunos lineamientos sobre la conformación de la Administración del Estado digital y su relación con las personas a través de tecnologías de la información.

1. Documento elaborado por Danielle Zaror Miralles con las contribuciones de los profesores Daniel Álvarez Valenzuela, Alberto Cerda Silva y Rodrigo Moya García y de los investigadores e investigadoras Michelle Bordachar Benoit, Paloma Herrera Carpintero, Juan Pablo Hernández Hellriegel, Daniela Olivares Rojas, Pablo Trigo Kramcsák, Rodrigo Vargas Acosta y Eduardo Vilches Fuentes.

2. Ley 21.20o que modifica el capítulo XV de la Constitución Política de la República. 


\section{Derechos digitales para una nueva constitución}

En el ámbito de los derechos y libertades fundamentales creemos necesario recoger la experiencia constitucional chilena que tiene más de doscientos años de trayectoria y evolución, e incorporar en la formulación de algunos de estos derechos y libertades ciertos elementos que permitan un mejor desempeño de las nuevas formas de ejercicio y una mayor eficacia frente a las amenazas que pueden surgir por el uso de tecnologías digitales. Asimismo, creemos necesario incorporar nuevos derechos y libertades al catálogo que establezca el nuevo texto constitucional.

En los siguientes párrafos revisaremos algunos de estos derechos y libertades, comenzando por aquellos que ya cuentan con algún nivel de reconocimiento constitucional en el texto vigente.

1. Derecho a la privacidad. La Constitución hoy consagra el «respeto y protección de la vida privada y a la honra de la persona y de su familia». Este derecho, que tradicionalmente ha sido concebido como un derecho de carácter individual, está evolucionando hacia una dimensión colectiva. La protección individual de esta dimensión de las personas resulta parcialmente eficaz para una vida análoga y crecientemente ineficaz a la luz del uso intensivo de tecnologías digitales.

La recolección - muchas veces indebida - de grandes volúmenes de datos personales en cada una de las interacciones digitales, hace que su abordaje deba ser analizado no solo desde el impacto en el individuo, sino también desde sus repercusiones políticas, sociales, comunitarias y, en última línea, considerando los efectos en la democracia.

Hay autoras que estiman que existiría una dimensión pública de la privacidad $y$, en ese entendido, su protección se vuelve un asunto de interés colectivo por la codependencia de la privacidad de cada uno, es decir, la intensidad de la privacidad de uno estaría estrechamente ligada al contexto y decisiones del colectivo al que se pertenece (Véliz, 202ob; Zuboff, 2020).

Así comprendida, la privacidad se parecería más, en el nuevo contexto, al derecho a vivir en un medio ambiente libre de contaminación. En este sentido, no basta con que se realicen acciones individuales para gestionar la privacidad cuando el sistema completo no está diseñado o estructurado ni organizado para proteger la privacidad como un todo. Esto, por supuesto, supone cambiar la posición desde la que se piensa el derecho a la privacidad, ya no como una manifestación de autonomía privada, sino como un sistema de intromisiones y accesos indebidos que generan impactos en el colectivo. ${ }^{3}$ La privacidad es importante tanto para el individuo como para la sociedad y los arreglos institucionales a través de los cuales se proteja deben estar culturalmente ligados al lugar desde donde se hace esta reflexión (Véliz, 2020a).

3. Daniel Innerarity, «Nada personal», La Vanguardia, 16 de enero de 2021, disponible en https://bit. ly/3xpY6Ny. 
Otra dimensión de la privacidad es su concepción como expresión de autonomía y autodeterminación personal, en especial, en el ámbito de las decisiones sobre su propio cuerpo y su identidad. Este es un aspecto que en Chile ha tenido escaso desarrollo doctrinario y jurisprudencial (Figueroa, 2014; Peña González, 2001; Undurraga, 2005) y que debiera ser revisado en el debate constitucional.

También la privacidad debe ser analizada como respuesta a la vigilancia estatal. Como es bien sabido, el desarrollo tecnológico ha impactado en los sistemas de vigilancia y ha hecho proliferar la instalación de cámaras de videovigilancia, drones, sistemas de reconocimiento facial, controles automatizados en las vías públicas, todo ello con finalidades declaradas de seguridad pública y, supuestamente, de mejora en la persecución penal. En este sentido, se debiera comprender el derecho a la privacidad como límite al ejercicio de las facultades punitivas y de control social del Estado.

Finalmente, se debe discutir el derecho a la privacidad como respuesta al perfilamiento. El crecimiento sin límites de grandes conglomerados tecnológicos constituye una gran amenaza para la privacidad, por cuanto el gran volumen de información que se recoge sobre las personas hace posible que se defina o caracterice a los usuarios de formas en que la historia no tiene registros. Esta situación arrincona «el derecho a ser dejado solo», y también hace que emerjan otras dimensiones de este derecho, como el derecho a la desconexión o el derecho a no ser perfilado.

En resumen, en la sociedad digital la privacidad, entendida en su aspecto activo y pasivo, se ve fuertemente amenazada, tanto por actores privados como estatales, lo que requiere revisitar y redoblar los esfuerzos en torno a su comprensión y protección.

2. Derecho a la protección de datos personales. Se trata de un derecho fundamental recientemente reconocido en Chile cuya mantención en el nuevo texto constitucional debería ser un punto de partida para robustecer su ejercicio y protección, pero fortaleciendo su dimensión de exigibilidad y amparo efectivo a través de la creación de una autoridad de protección de datos personales. En este sentido, considerar el derecho a la autodeterminación informativa en la nueva constitución es imprescindible si tenemos presente, por una parte, el uso intensivo de datos personales en las actividades cotidianas de las personas e instituciones, y, por otra, el uso a gran escala de múltiples análisis predictivos, masivos, invasivos y potencialmente discriminatorios que emplean datos personales, en donde, además, la afectación que se produce es también colectiva.

3. Derecho a la libertad de expresión. Definir qué es hoy la libertad de expresión es un desafío en curso. No es un derecho distinto si lo abordamos desde un entorno análogo, pero no es el mismo derecho cuando se expresa en un entorno digital. Su contenido y límites (principalmente aquellos que resultan del ejercicio de otros derechos) parecieran ser parte de las respuestas que debe entregar la nueva redacción constitucional. 
Un derecho que respondió a las necesidades del siglo XVIII debe ser reconstruido teniendo en cuenta el contexto social y tecnológico del siglo XXI. Es necesario reflexionar sobre el objetivo que debe cumplir en un entorno como el digital. La acción, por ejemplo, no está cubierta por la libertad de expresión, y en los entornos digitales buena parte de la expresión de ciertas ideas puede derivar en acciones perjudiciales para la vida y la integridad física y psíquica de las personas y también de la democracia. Por ello, resulta necesario discutir si la Constitución está dispuesta a extender su protección a la expresión divulgada por «cualquier medio» y si la masividad, ubicuidad e impacto serán pasadas por alto.

Sin perjuicio de lo anterior, se debe sumar a la reflexión el vínculo estrecho que hay entre la conectividad y la consecuente libertad o posibilidad de expresar ideas. Este documento busca hacer presente que se trata de un derecho en expansión y en tensión con la forma en que las tecnologías digitales permiten su ejercicio.

4. Derecho a la inviolabilidad de las comunicaciones privadas. Si bien el derecho a la inviolabilidad de las comunicaciones privadas es un derecho neutro tecnológicamente, de manera que se aplica sin distinciones tanto a comunicaciones analógicas como digitales (Álvarez-Valenzuela, 2019), en los últimos años han existido situaciones en Chile en que mecanismos de vigilancia masiva han implicado una afectación indebida de esta garantía (Malamud, 2018).

Asimismo, deben considerarse en el debate digital los nuevos escenarios digitales que dificultan la efectiva protección de las comunicaciones privadas, como el uso de servidores y casillas de correo electrónico controladas o accesibles (desde el punto de vista técnico) por terceros (por ejemplo, casillas institucionales o proporcionadas en el ámbito laboral), el almacenamiento en la nube, y el tratamiento y retención de los metadatos de esas comunicaciones para finalidades distintas a la privada.

5. Inviolabilidad del hogar y de los documentos privados. Teniendo presente que la garantía de inviolabilidad protege los recintos privados, en una concepción amplia del hogar, se debe discutir su aplicabilidad a los denominados recintos electrónicos, como servidores, cloud computing, entre otros, tanto de intromisiones físicas como remotas.

6. Propiedad intelectual y acceso al conocimiento. En el artículo 19 núm. 25 de la Constitución se reconocen los derechos de autor, en su dimensión patrimonial y moral, así como los derechos de propiedad industrial, conforme lo regule la ley, y remitiendo a las normas constitucionales relativas al derecho a la propiedad. Se trata de una regulación con enfoque propietario. Asimismo, se reconoce la libertad de crear y difundir las artes, aunque no se desarrolla mayormente esta dimensión del derecho.

El debate constitucional puede ser una oportunidad para ampliar la forma de concebir la propiedad intelectual y los derechos de autor como derechos de doble dimensión (Álvarez-Valenzuela y Correa Pérez, 2016), que junto con proteger los intereses subjetivos de titulares, autores y autoras, garantice la dimensión colectiva 
de acceso a la cultura y de acceso al resultado del progreso tecnológico, en línea con la Constitución estadounidense (en la llamada «cláusula del progreso», que señala que el Congreso, para la promoción de las artes y las ciencias, podrá establecer leyes de derechos exclusivos por tiempo limitado) y con la Declaración Universal de los Derechos Humanos.

Reconocer una norma de estas características tiene al menos dos beneficios. Por un lado, la dimensión de acceso en los derechos de autor podría suponer el avance hacia un sistema de excepciones amplias a nivel legal, en la medida en que una protección demasiado expansiva de los derechos de autor limita los derechos del público de acceder y crear nuevas obras. Por otro lado, abre la puerta para todas las políticas públicas que busquen equidad social a través de la expansión del progreso científico, como lo es por excelencia el progreso tecnológico.

\section{Derechos de la sociedad de la información: nuevos ámbitos}

7. Regla constitucional sobre espectro radioeléctrico. Se trata de una materia que resulta elemental para la discusión de cualquier otro derecho en la sociedad digital. La distribución justa y equitativa del espectro radioeléctrico impacta en la cobertura y calidad de la conectividad de todo el territorio de un país, así como en la capacidad de respuesta frente a desastres naturales porque asegura la estabilidad de la infraestructura crítica. El debate y su correlativa incorporación constitucional puede garantizar que su distribución responda a lógicas de equidad social y que consideren en su operación el mayor y mejor bienestar social posible.

8. Derecho a la conectividad. Se trata de uno de los temas más debatidos durante la pandemia. Existe un aparente consenso en la industria y en la sociedad civil sobre su relevancia para el aseguramiento de otros derechos fundamentales (Miranda, 2016), por ejemplo, la libertad de trabajo, el derecho a la educación, el derecho de propiedad, el derecho a la protección de la salud, la libertad de emitir opinión y la de informar, etcétera. Su núcleo de contenido debiera estar formado por todos aquellos eslabones necesarios para que una persona se pueda conectar. Cuando se habla de capacidad de acceder a internet (la posibilidad que se tiene de llegar a internet), conectividad (el acceso de manera directa), alfabetización digital (la apropiación de los contenidos) y calidad.

Resulta prioritario que el acceso a la conectividad se consagre como derecho social, considerando que resulta indispensable no solo para el ejercicio de derechos fundamentales, sino para que el Estado pueda materializar a su vez otros objetivos necesarios para el cumplimiento de sus fines $;$.

Lo interesante es que este derecho de acceso a internet se ha planteado como un estándar vinculado al efectivo ejercicio de la libertad de expresión, lo que la sociedad ya se encuentra en posición de garantizar. 
9. Identidad digital. El derecho a la identidad se considera un derecho humano. Si bien las nuevas tecnologías pueden ayudar a garantizar este derecho a una escala mucho mayor que antes, la creación de una identidad digital presenta riesgos como la marginación de las personas que no tienen acceso a la tecnología y la intensificación de la vigilancia y control sobre los individuos (máxime si se emplean modalidades biométricas).

Muchos países de la región (México, Paraguay, Perú, Uruguay, Costa Rica, etcétera) se encuentran implementando modelos de identidad digital. La doctrina jurídica nacional prácticamente no ha reflexionado sobre su impacto, sus principios o sus reglas, lo que es una verdadera brecha considerando que en muchos países se habla hace tiempo de una identidad digital legal, es decir, un identificador que permite una interacción con ciertos prestadores estatales o privados que excluyen el anonimato y obligan al uso de claves de autenticación para acceder a servicios personales e intransferibles (por ejemplo, salud, finanzas, prestaciones sociales, etcétera). En definitiva, la seguridad de estas plataformas descansa en ecosistemas regulatorios comprehensivos, que permiten su uso aplicando salvaguardas adecuadas, con un enfoque en las personas que reciben servicios por parte del Estado. Además, requieren una declaración de responsabilidad por parte del Estado porque los riesgos recaen en el ciudadano.

10. Ciberseguridad digital. Una dimensión correlativa de la ciudadanía digital, en los términos en que lo hemos evidenciado, requiere que se reconozcan mecanismos que preserven esta dimensión de su integridad y aumenten la confianza de los ciudadanos en el ciberespacio, sobre todo considerando la creciente digitalización de la vida y la economía (Pareja y otros, 2017). Una parte de la discusión gira en torno a la gestión de una identidad en forma segura de los individuos, pero el tema también puede abarcar al Estado completo, ya sea por la afectación de las infraestructuras tecnológicas, los componentes lógicos de la información y las interacciones que se verifican en el ciberespacio, debido a amenazas de diversa naturaleza, ya sean actividades hostiles de terceros países o la actividad de organizaciones criminales. Resulta indispensable que la nueva regulación constitucional mandate la creación de organismos que pueden entregar respuestas coordinadas al más alto nivel. En ese sentido, la delegación legal para su institución y atribuciones podría resolver el asunto conforme a los cambios que vaya experimentando este sector.

\section{Administración del Estado}

Hay voces que plantean la necesidad de asegurar una continuidad digital de los servicios del Estado (derecho al relacionamiento digital). Esto sin duda tiene impactos en todos los derechos anteriores. La transformación digital trae como consecuencia el riesgo de paralización de los servicios del Estado, lo que se puede traducir en la 
cesación de muchas prestaciones públicas, desde servicios básicos hasta la gestión de información en la entrega prestaciones sociales. En la medida en que la transformación digital abarca otros poderes, como el judicial, el asunto se vuelve mucho más crítico pues en caso de paralización, o cualquier otro evento que impida la prestación de servicios públicos, será mayor el número de garantías constitucionales afectadas.

Este aspecto está en directa relación, por un lado, con el acceso a internet —en la medida en que más personas tengan acceso a una conexión estable y económica, mayor será el grupo beneficiado y más serán los servicios que pueda ofrecer el Estado-, y por otro, con la inclusión digital, esto es, con la capacidad del Estado de transmitir a la ciudadanía la existencia de estos servicios digitales y que las personas cuenten con la debida alfabetización para poder entender e interactuar con este Estado digital. La inclusión como concepto se concibe dentro de un campo bastante más amplio, pero esta es una de las áreas que debe ser considerada para dotar al concepto de un núcleo de contenido (Ribeiro Rosa, 2013). Así, por ejemplo, la igualdad ante la ley vería modificada y ampliada su dimensión a la luz de este nuevo fenómeno.

Esto resulta relevante cuando el Estado invisibiliza su responsabilidad en la prestación y continuidad de sus servicios, así como cuando no establece mecanismos que permitan a la ciudadanía reclamar cuando ha sido objeto de decisiones automatizadas que tienen consecuencias injustas. Además, no debe perderse de vista que el Estado en sus actuaciones puede cometer discriminaciones, por lo que el diseño de una norma constitucional que reconozca un Estado digital debe establecer mecanismos que permitan corregir las vulneraciones que se produzcan y ofrecer la transparencia suficiente para detectar cuando surjan.

En este sentido, la consagración del derecho de acceso a la información debe rediseñarse pensando en que las personas tengan derecho a saber qué tecnología se está usando en el Estado y las garantías para poder conocer los impactos sobre sus vidas.

En relación con el ejercicio de peticiones a la autoridad, es imprescindible considerar la no discriminación. Resulta evidente que se han modificado sustancialmente los medios de interacción en el contexto del gobierno electrónico, y ello, a su vez, ha reconfigurado las formas de relacionarse. Lo más importante es el empoderamiento ciudadano.

La Administración del Estado ha estimulado la utilización de medios tecnológicos, ya sea como medio para presentar peticiones a la autoridad, como canal de comunicación efectivo y/o elevándolo a mecanismo capaz de proporcionar información y servicios a la ciudadanía (transaccional). Dichas políticas pueden ser tremendamente beneficiosas desde la óptica del reforzamiento de los derechos ciudadanos, pero también peligrosas si dejan a parte importante de la población fuera de un espacio virtual de interacción y con ello afectan su libre e igualitario ejercicio. Dejar al margen de estos canales de comunicación a personas que, por su condición económica, nivel de alfabetización digital o emplazamiento geográfico no pueden acceder 
fácilmente, significa vulnerar el derecho a presentar peticiones a la autoridad y, con ello, a permitir el normal ejercicio de la garantía por medios electrónicos.

\section{Referencias}

Álvarez-Valenzuela, Daniel (2019). La inviolabilidad de las comunicaciones privadas electrónicas. Santiago: Lom.

Álvarez-Valenzuela, Daniel y Marco Correa Pérez (2016). «La doble dimensión del derecho de autor: El acceso a la cultura y los derechos de autor». Revista de Derecho Público, 85: 11-32. DOI: 10.5354/0719-5249.2016.44958.

FigueroA, Rodolfo (2014). Privacidad. Santiago: Ediciones Universidad Diego Portales.

Malamud, Samuel (2018). «Videovigilancia y privacidad. Consideraciones en torno a los casos Globos y Drones». Revista Chilena de Derecho y Tecnología, 7 (2): 137162. DOI: 10.5354/0719-2584.2018.49097.

Miranda Bonilla, Haideer (2016). «El acceso a internet como derecho fundamental». Revista Jurídica IUS Doctrina, 9 (5): 1-23. Disponible en https://bit.ly/3yvDSIS.

Pareja, Alejandro, Mari Pedak, Carlos Gómez y Alejandro Barros (2017). La gestión de la identidad y su impacto en la economía digital. Documento para Discusión núm. IDB-DP-529. Iter-American Development Bank. DOI: 10.18235/00o0786.

Peña González, Carlos (2001). «Notas sobre las relaciones entre privacidad y autonomía». Revista de Derecho y Humanidades, 8. Disponible en https://bit. ly/2UuwAA8.

Ribeiro Rosa, Fernanda (2013). «Inclusión digital como política púlica: Disputas en el campo de los derechos humanos». Sur. Revista Internacional de Derechos Humanos, 2013: 33-55. Disponible en https://www.corteidh.or.cr/tablas/r32486-1.pdf.

Undurraga, Verónica. (2005). «La privacidad como bien jurídico». En Estudios de Derecho Civil. Jornadas nacionales de Derecho Civil, Valdivia (pp. 509-530). Santiago: Lexis Nexis.

VéLIz, Carissa (2020a). Data, privacy and the individual. Privacy matters. Center for the Governance of Change. Disponible en https://bit.ly/3wjo8kh.

-. (2020b). Privacy is power. Bantam Press.

Zuboff, Shoshana (2020). La era del capitalismo de la vigilancia: La lucha por un futuro humano frente a las nuevas fronteras del poder. Barcelona: Paidós. 


\title{
REVISTA CHILENA DE DERECHO Y TECNOLOGÍA
}

La Revista de Chilena de Derecho y Tecnología es una publicación académica semestral del Centro de Estudios en Derecho Informático de la Facultad de Derecho de la Universidad de Chile, que tiene por objeto difundir en la comunidad jurídica los elementos necesarios para analizar y comprender los alcances y efectos que el desarrollo tecnológico y cultural han producido en la sociedad, especialmente su impacto en la ciencia jurídica.

\author{
EDITOR GENERAL \\ Daniel Álvarez Valenzuela \\ (dalvarez@derecho.uchile.cl) \\ SITIO WEB \\ rchdt.uchile.cl \\ CORREO ELECTRÓNICO \\ rchdt@derecho.uchile.cl \\ LICENCIA DE ESTE ARTÍCULO \\ Creative Commons Atribución Compartir Igual 4.o Internacional
}

\begin{abstract}
La edición de textos, el diseño editorial
y la conversión a formatos electrónicos de este artículo

estuvieron a cargo de Tipográfica

(www.tipografica.io).
\end{abstract}

